Please do not remove this page

RMIT

UNIVERSITY

\title{
Multichannel vector sum phase shifter
}

Bui, Lam; Ghorbani, Kamran; Mitchell, Arnan

https://researchrepository.rmit.edu.au/esploro/outputs/9921859953001341/filesAndLinks?institution=61RMIT_INST\&index=null

Bui, L., Ghorbani, K., \& Mitchell, A. (2006). Multichannel vector sum phase shifter. Optics Letters, 31(5), 577-579. https://doi.org/10.1364/OL.31.000577

Published Version: https://doi.org/10.1364/OL.31.000577

Repository homepage: https://researchrepository.rmit.edu.au

(c) 2006 Optical Society of America

Downloaded On 2023/04/26 22:28:29 +1000

Please do not remove this page 


\title{
Multi-channel vector sum phase shifter
}

\author{
Lam Bui, Kamran Ghorbani, Arnan Mitchell \\ RMIT University, School of Electrical and Computer Engineering, \\ GPO Box 2476V, Melbourne 3001, Australia
}

Compiled September 22, 2005

\begin{abstract}
This letter demonstrates a novel multi channel vector sum phase shifter that is suitable for phased array antenna applications. Each channel is implemented using a distinct optical wavelength. Selective control of each channel is performed using an acousto-optic polarisation coupler. The concept is successfully demonstrated for two individually controlled channels. For each channel, a continuously variable frequency-linear phase shift is demonstrated between DC and $7 \mathrm{GHz}$ with the phasing range exceeding 100 degrees. (C) 2005 Optical Society of America

OCIS codes: $060.2340,070.6020,280.5110,350.4010$
\end{abstract}

Modern electronic warfare radars operate over a wide scanning angle and frequency band! 1 These radars often employ phased array antenna (PAA) technology ${ }^{2]} \mathrm{A}$ PAA consists of many antenna elements whose phase excitations can be individually controlled. For broadband PAAs, time shifters or true time delay techniques (TTD) are required $!^{3}$ Over the past several years, photonics has been actively investigated for TTD systems due to its broad bandwidth, immunity to EMI and light weight! 4 The drawbacks of these systems however, are their complexity and high component and installation costs.

For narrow band applications, the vector sum technique is a well known phase shifting scheme. ${ }^{56}$ We have demonstrated this scheme over a broad frequency range to approximate TTD characteristics, ${ }^{7}$ hence conceiving a vector sum phase shifter (VSPS) as a cost effective solution for TTD PAAs.

Several realisations of the VSPS have been demonstrated. These include using a variable coupler and two optical delay lines 7 as well as using polarisation diversity and polarisation group delay in a length of high birefringence fibre ${ }^{8}$ Among the two implementations, the polarisation diversity technique is the most elegant. Recently, we have demonstrated a rapidly tunable VSPS employing an acousto-optic polarisation coupler (AOPC), hence it is called acousto-optic VSPS (AO-VSPS)

For PAA applications, each antenna element requires a dedicated phase shifter. Many phase shifters are thus employed. The proliferation of phase shifters would result in increasing system cost and bulk. It is thus advantageous to invent a phasing device that could serve several antenna elements. It is proposed that several wavelength channels could be independently controlled using a single AO-VSPS device. In this letter, we verify this concept by demonstrating the dual channel operation of the AO-VSPS.

The experimental setup to demonstrate dual channel VSPS is presented in Figure 11 The VSPS consists of an AOPC and a $36 \mathrm{~m}$ Fujikura PANDA fibre. This fibre length produces a differential time delay between two polarisation modes of $47.6 \mathrm{ps}$ (assuming a fibre bire- fringence of $\left.39.6 \times 10^{-5}\right)$. Since the fibre birefringence is small, several centimeters of error in the fibre length translates to a negligible time delay error. This tolerance significantly improves the device manufacturability.

The first channel of the VSPS was implemented as explained in a previous publication. ${ }^{9}$ To introduce a second channel, an additional laser, a Mach Zehnder modulator (MZM) and a photo-detector were added. The two channels were multiplexed and then demultiplexed using a 3 $\mathrm{dB}$ coupler and an arrayed waveguide grating (AWG) respectively. A polarisation beam splitter (PBS) was employed to ensure that a known polarisation (TM in this case) was launched into the AOPC. Polarisation controllers PC1 and PC2 were inserted between the MZMs and the WDM multiplexer to transform the unknown polarisations at the MZM single mode fibre outputs to the required polarisation (TM) of the AOPC input. For PAA applications, the relative phase shifts between channels are important. At zero phase settings, the two channels should produce identical phase shifts. The two channels were thus calibrated to have identical electrical lengths using microwave trombone lines TL1 and TL2 when the AOPC was inactive (no acoustic power).

The major component of the Figure 1 setup is the AOPC. Fundamentally, the AOPC is a wavelength selective polarisation coupler!10 Polarisation conversion occurs at optical wavelengths that are phase matched with the acoustic frequencies. By varying the acoustic wave power, the ratio of optical power in each polarisation can be continuously adjusted.

The AOPC was designed and fabricated at the Microelectronics and Materials Technology Center (MMTC) at RMIT University. Before being utilised in the experimental setup of Figure 1 1 the AOPC was characterised. Figure 2 presents the measured polarisation transfer function of the AOPC when an acoustic wave of $170.65 \mathrm{MHz}$ was used. It is evident from Figure 2 a) that phase matching occurs at $1560 \mathrm{~nm}$. There is a $2 \mathrm{~dB}$ difference in power between TE and TM modes. This is due to the polarisation dependent loss of the unoptimised optical waveguide used for the AOPC fabrication. From 


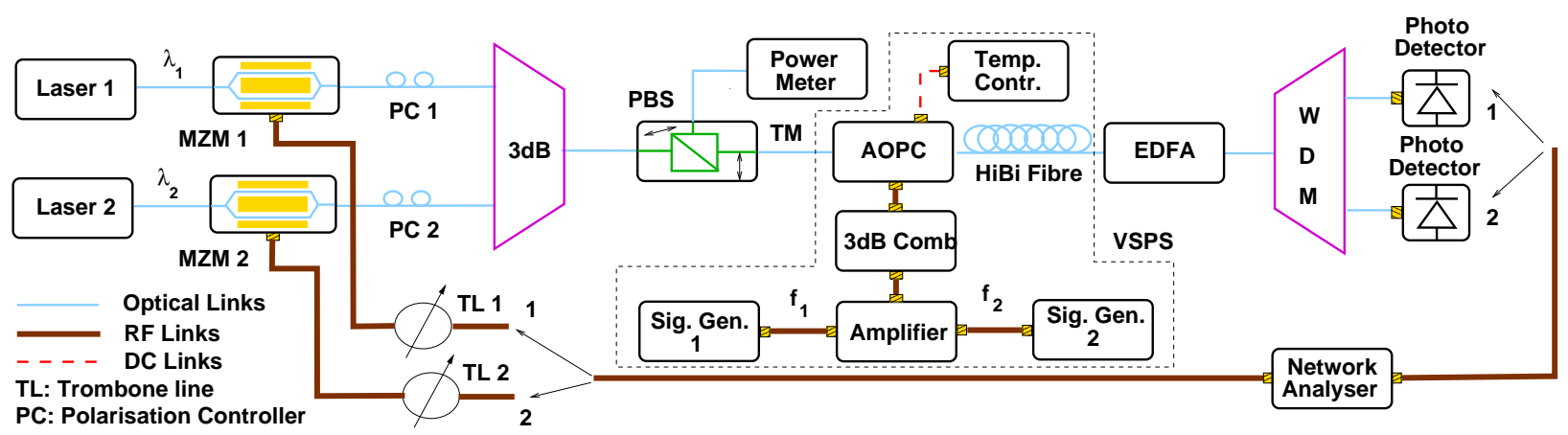

Fig. 1. Experimental setup to demonstrate dual channel VSPS.
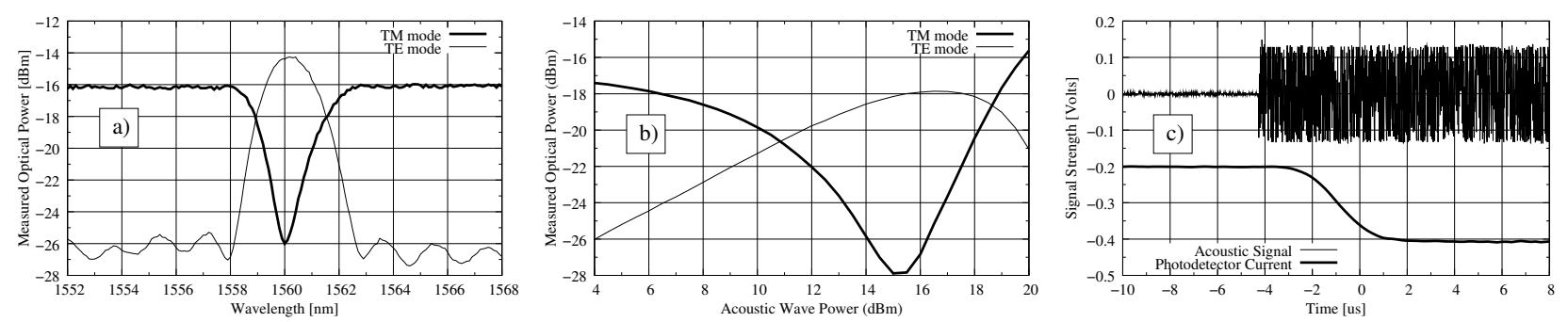

Fig. 2. Measured characteristics of the AOPC. a) Wavelength transfer characteristic, b) Polarisation coupling characteristic, c) Transient characteristic.

Figure 2 b), polarisation coupling depends on the acoustic power level with a maximum extinction ratio of $10 \mathrm{~dB}$ observed at $15 \mathrm{dBm}$ power level. To determine, how fast light can be switched between polarisations, the temporal response was measured by turning the acoustic power on and off. Figure 2 c) presents the driving acoustic waveform and the AOPC configuration time from TE polarisation to TM polarisation. The measured time offset between the acoustic transition and the polarisation switching is less than $5 \mu \mathrm{s}$. The AOPC is thus a wavelength selectable polarisation coupler with a configuration time in the order of $5 \mu \mathrm{s}$.

Evidently the AOPC rotates light polarisation for a narrow wavelength range that phase matches with the acoustic frequency. It has been demonstrated that using multiple acoustic frequencies simultaneously will result in polarisation rotation of multiple wavelengths. ${ }^{11}$ It is useful to characterise these rotations when operating the AOPC under multiple acoustic frequencies. Two acoustic waves at frequencies of $171.68 \mathrm{MHz}$ and $172.1 \mathrm{MHz}$, each with a power level of $13 \mathrm{dBm}$, were used. Figure 3 presents the measured output power in TM polarisation for TM input light over a bandwidth of $12 \mathrm{~nm}$.

It is observed from Figure 3 that only when a single acoustic wave is used, polarisation rotation happens at $1551.5 \mathrm{~nm}$ (for $171.68 \mathrm{MHz}$ ) and $1548.5 \mathrm{~nm}$ (for 172.1 $\mathrm{MHz}$ ). When two acoustic waves are launched simultaneously, polarisation rotations shift to slightly shorter wavelengths. This could be attributed to the heating of the device due to additional acoustic power. Nevertheless, the change in response is within $1 \mathrm{~dB}$ and the

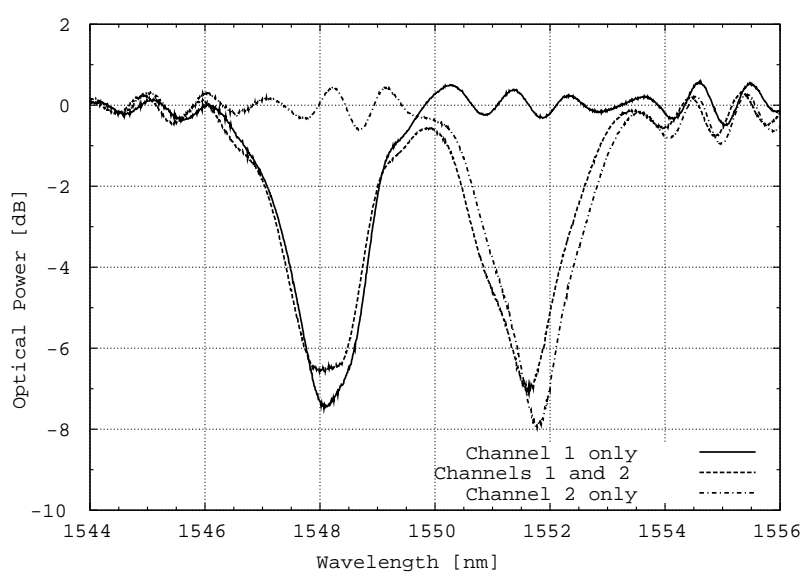

Fig. 3. Measured TE coupling characteristic of the AOPC under the presence of single and both acoustic waves.

wavelength shift is less than $0.5 \mathrm{~nm}$. It is therefore concluded that the performance of the device is sufficient for demonstrating dual channel operation of the VSPS.

Having characterised the AOPC, the experiment was set up as shown in Figure1 The response of each channel was measured using a $20 \mathrm{GHz}$ network analyser (NA). Initially, the NA was calibrated with respect to the response of channel 1 when the AOPC was inactive. With the absence of acoustic wave 2 , the power of acoustic wave 1 was then increased and the response of channel 1 was measured. Figures 4 a) and b) present these measurements. It is evident from Figure 4 a) that the 

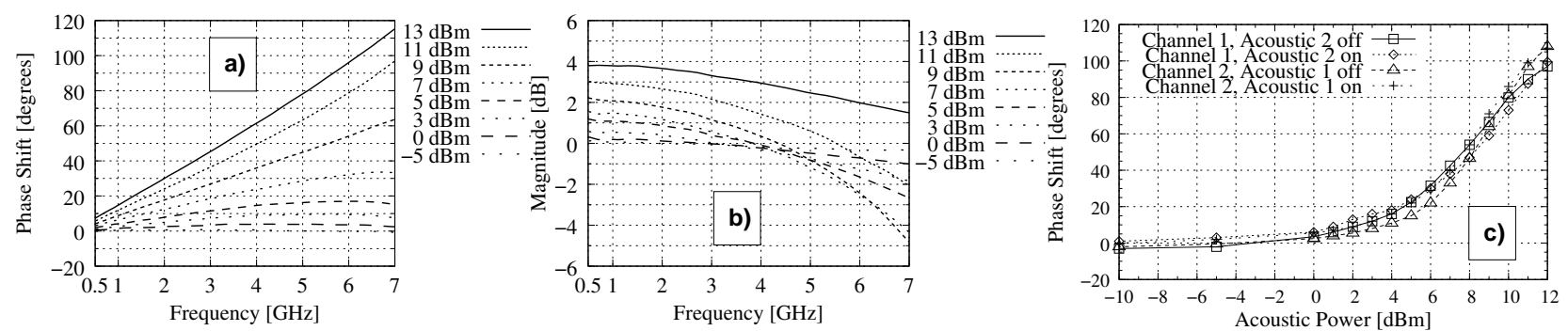

Fig. 4. Measured responses of the dual channel VSPS. a) Phase response of channel 1, b) Magnitude response of channel 1, c) Phase responses at $7 \mathrm{GHz}$ of channels 1 and 2 under four measured scenarios.

phase response is linear with frequency and all project to zero phase at DC. A maximum phase shift of over 100 degrees was obtained when an acoustic power level of $13 \mathrm{dBm}$ was used. The magnitude response degrades toward higher frequencies due to resonant characteristics of the VSPS! $]^{[7}$ At low frequencies, there is about $4 \mathrm{~dB}$ variation in the amplitude response. It is important to note that this low frequency amplitude degradation is not intrinsic to the VSPS but is attributed to a defect in the AOPC that was used. This AOPC exhibits a $2 \mathrm{~dB}$ polarisation dependent loss as observed in Figure 2 a).

Similar measurements of channel 1 were obtained with the presence of the $13 \mathrm{dBm}$ acoustic wave 2 . Channel 2 was then measured with the absence and then with the presence of the $13 \mathrm{dBm}$ acoustic wave 1. Figure 4 c) presents the phase responses of channel 1 and channel 2 for the four measurement scenarios. It is evident that the responses are very similar, suggesting that channel 1 and channel 2 could be individually and simultaneously controlled. The discrepancies among the phase responses indicate the effect of channel interaction as observed previously in Figure 3. This effect could be reduced by improving the wavelength selectivity of the AOPC or having more efficient device thermal control or simply increasing the channel separation.

There are several factors that limit the total number of channels. The minimum channel separation is limited by the channel interaction discussed previously. The available bandwidth is limited by the EDFA to $1530-1560$ $\mathrm{nm}$ range. With the increasing number of channels, the acoustic power also increases accordingly. It is anticipated that the ultimate number of channels would be limited by the threshold acoustic power at which the AOPC becomes unstable due to the thermal effects. Issues associated with the acoustic wave generation and the temperature control become significant for a large number of channels and need to be addressed to make the device practical.

In conclusion, we have demonstrated a dual channel VSPS. Each of these channels can be independently and simultaneously manipulated. Additional wavelengths can be introduced to extend the device capacity to many phasing channels. The number of channels will be limited by wavelength interaction and the thermal instability of the AOPC. It is also proposed that several VSPSs should be cascaded to increase the channel phase shift. Integration of the VSPS into a practical phased array antenna and demonstration of beam steering are currently being investigated.

\section{References}

1. J. C. Veihl, D. M. Hodges, and C. Monzon, "Reconfigurable aperture decade bandwidth array," in IEEE Inter. Symp. on Antennas and Propagation, 2000 (2000).

2. R. Axford, R. Major, and J. Rockway, "An assessment of multi-function phased array antennas for modern military platforms," in IEEE Inter. Symp. on Phased Array Systems and Technology, 2003, pp. 365 - 370 (2003).

3. J. Frank, "Bandwidth criteria for phased array antennas," in Proceeding of phased array antenna symposium, Polytechnique Institute of Brooklyn, Brooklyn, USA, pp. 243-253 (1970).

4. A. N. Riza and J. B. Thompson, Selected papers on photonic control systems for phased array antennas (SPIE Optical Eng. Press, 1997).

5. S. J. Kim and N. H. Myung, "A new active phase shifter using a vector sum method," IEEE Microwave Guided Wave Lett. 10(6), 233-235 (2000).

6. K.-H. Lee, Y. M. Jhon, and W.-Y. Choi, "Photonic phase shifters based on a vector-sum technique with polarization-maintaining fibers," Opt. Lett. 30(7), 702704 (2005).

7. L. A. Bui, A. Mitchell, K. Ghorbani, and T. H. Chio, "Wideband RF photonic vector sum phase shifter," Electron. Lett. 39(39), 536-537 (2003).

8. L. A. Bui, A. Mitchell, and T. H. Chio, "Wideband vector sum phase shifter with minimal coherent interference," in COIN 2003/the 28 $8^{\text {th }}$ ACOFT, Melbourne, Australia, pp. 645-648 (2003).

9. L. A. Bui, A. Mitchell, K. Ghorbani, and T. H. Chio, "Electronically tunable vector sum phase shifter using acousto optic polarisation coupler," in BGPP 2005/30 ACOFT Conference, Sydney, Australia (2005).

10. H. Mendis, A. Mitchell, I. Belski, M. Austin, and O. A. Peverini, "Design, realisation and analysis of an apodised, film-loaded acousto-optic tunable filter," "Appl. Phys. B 73, 489-493 (2001).

11. K. W. Cheung, D. A. Smith, J. E. Baran, and B. L. Heffner, "Multiple channel operation of integrated acousto-optic tunable filter," Electron. Lett. 25(6), 375376 (1989). 